\title{
Energy efficient scheme to Jointly Optimize Coverage and Connectivity in Large Scale Wireless Sensor Network
}

\author{
Deepak S. Sakkari *, T.G. Basavaraju** \\ *JNTUH, Hyderabad, India \\ ** Departement of CSE, Government SKSJTI, Bangalore, India
}

\begin{abstract}
Article Info
Article history:

ABSTRACT

Received Feb 9, 2015

Revised Mar 19, 2015

Accepted Apr 16, 2015

\section{Keyword:}

Connectivity

Coverage

Energy Efficiency

Efficient coverage and connectivity are two important factors that ensure better service quality especially during tracking targets or monitoring events in wireless sensor network. Although massive amount of studies has been carried out in the past to enhance coverage and connectivity issues, till date very few studies have witnessed a significant and standard outcomes that can opt further. Hence, this paper introduces a computationally efficient technique for jointly addressing both coverage and connectivity problems in large-scale wireless sensor network that ensures optimal network lifetime too. The proposed system has been empirically designed, and algorithms formulated to ensure energy efficient monitoring of event. The outcomes of the study are compared with standard energy efficient hierarchical protocol to benchmark the results.
\end{abstract}

Optimization

Wireless Sensor Network
Copyright (C) 2015 Institute of Advanced Engineering and Science. All rights reserved.

\section{Corresponding Author:}

Deepak S. Sakkari,

Research Scholar,

Jawaharlal Nehru Technological University,

Hyderabad, India.

Email: deepaksakkari@gmail.com

\section{INTRODUCTION}

The area of wireless sensor network has received quite a lot attention from the research community in last ten years owing to the advantages as well as spontaneously evolving challenges in the technology. A wireless sensor network comprises of the group of sensor nodes that are either positioned in a unique location (uniform) or distributed arbitrarily in the environment (random). The sensor nodes are small electronic devices that have the capability to sense certain physical attributes like moisture, heat, motion, pressure, smoke, etc. Right from habitat monitoring to healthcare application, wireless sensor network has found its applicability in multiple commercial needs [1]. A closer look at the operation of wireless sensor network finds that there are three types of sensor nodes e.g. member sensor nodes, cluster head, and base station. The member sensor node gathers the raw physical data and transmits to the cluster head. It is said that clustered usually possess higher residual energy compared to member nodes. The cluster head is required to possess such high residual energy as they are mainly responsible for transmitting massively aggregated data to the sink. This phenomenon is termed as data aggregation [2]. Hence, in order to visualize the effective data aggregation phenomenon, it is essential that all the nodes retain maximum energy and assists in forwarding non-redundant data to the base station. Hence, there are various internal as well as external factors that play a crucial role in data aggregation process. The internal parameters are routing protocols, battery lifetime, packet redundancies, security protocols, etc. while the external factors are interference, noise, scattering, channel fading, etc. In spite of such potential capabilities, wireless sensors suffer from certain issues e.g. restricted computational capability, less buffer, finite battery life, minimal resource availability. Owing to such characteristics, the performance of the network is highly affected where the prime causes is energy [3]. 
Apart from the issues discussed above, there are certain more prominent issues in wireless sensor network termed as coverage and connectivity issues, which is the major focus of the present paper. The term coverage is defined as the optimal sensing and transmission distance required by the sensor node to perform monitoring the event in a particular location [4]. Sometimes, coverage is also interpreted as criteria to ensure optimal service quality. The significance of the coverage issue is very prominent and very closely associated with the energy factor [5]. It was seen in many cases that reduction of battery life spontaneously reduces the coverage area and thereby adversely affect the quality of services. This may not be a big issue in small-scale network, but possess a challenging issue in large scale network. The second term connectivity is represented as the capability of the mote to ensure reachability to the sink at the time of data aggregation [6]. Hence, coverage and connectivity are very closely associated with each other and has potential affect in service quality, routing, and data aggregation. In case of non-availability of routes (poor connectivity), aggregated data doesn't reach sink and give rise to either packet drop or higher delay in the network.

This paper, therefore, studies various prior techniques and discusses the problems that have been identified from the review of the literature. The paper presents a simple and yet highly effective solution to address the significant issues of coverage and connectivity in wireless sensor network and ensure optimal energy preservation along with it. Section 2 discusses the prior research techniques followed by problem identification in Section 3. Section 4 discusses the proposed model, and Section 5 discusses about the implementation and result discussion. Finally, concluding remarks are being made in Section 6.

\section{RELATED WORK}

In the past decade, there has been enough work carried out towards mitigating connectivity and coverage issues in wireless sensor network. Our prior work [7] has already discussed couple of prior research attempts and excavated the research gap. This section discusses some more significant studies that have been investigated and studied to explore the better possibilities of coverage and connectivity in wireless sensor network. Zaidi et al. [8] have presented a framework where the author has adopted cost-based metrics to ensure efficient coverage in wireless sensor network. The authors have adopted two-dimensional deterministic based technique for the deployment as well as in random based deployment too. The outcome of the system was evaluated with respect to performance parameters e.g. probability of coverage in both one and two-dimensional area. However, the outcome was not found to be benchmarked. Agarwal et al. also continued discussion of efficiency in coverage and connectivity. [9] considering the problems of surveillance system. The author has adopted significant randomized technique using greedy technique where the simulation work is carried out using Monte Carlo approach for addressing the uniform coverage issues under finite sensing radius framework. The evaluation of the outcome was validated using gathered covered area without any comparative performance evaluation. The coverage factor was verified for Monte Carlo and Greedy techniques only. Similar line of research work was carried out by Bulut et al. [10] investigating the redundancy issues in coverage factor of wireless sensor network. The authors have adopted graph theory where the connectivity of the neighbor graph was the prime focus of the study. By adopting scheduling technique, the outcome of the study was evaluated using number of the active sensor nodes with specific QoS value without any performance comparative analysis. Beaudaux et al. [11] have investigated on k-Coverage problems in wireless sensor network. With an aid of layer-based localized algorithm, the framework provides enhanced capability with restricted routes with redundancies. The outcome of the system was evaluated using number of active nodes on WSNet simulator and was found not to be benchmarked. Wang et al. [12] have studied the coverage issues considering a unique application of sandstorm monitoring. The outcome of the study was evaluated using node density on the transmission range on various channel types. However, the outcome of the study was not found benchmarked. Kranakis et al. [13] have introduced a model to provide better range of the directional antenna on multiple hops. With an aid of performance parameters e.g. hop stretch factor, the outcome of the study was evaluated without any performance comparative analysis with other significant protocols. Stergiopoulos et al. [14] investigated on the coordination issues owing to the mobility of sensor nodes under constraints of RF communication in wireless sensor network. The authors have proposed a control technique to ensure optimal connectivity of the network, where the outcomes were evaluated using coverage performance factor on multiple samples. The outcome of the study still misses the benchmarking. Similar direction of the study considering mobility was also seen the work of Erdelj et al. [15]. However, this study was evaluated using percentage covering time as the performance factor in both circular and random route approach. Ren et al. [16] have formulated a maximization issue for coverage enhancement considering quality and connectivity as two essential parameters. Uniquely, the study also discusses with forecasting of energy fluctuation and outcomes were evaluated using coverage quality in both centralized and decentralized algorithms. Emphasis on energy efficient along with addressing coverage and connectivity issues was also seen in the work done by Noori and Rafeh [17]. The outcome of the study was 
evaluated with number of active nodes and coverage rate on NS2 simulator and compared with OGDC (Optimal Geographical Density Control) protocol [18]. Taymouri and Kangavan [19] have focused on the computational complexity of the investigating the connectivity tolerance in wireless sensor network. The authors have adopted both graph theory as well as mining techniques and tested on multiple distribution testbed. The outcome of the study was evaluated using connectivity tolerance. Hence, it can be seen that there are some potential studies being done considering the issues of coverage and connectivity, but a closer look at the prior work shows that much work is emphasized on coverage issues as compared to connectivity issues, and focus was much less towards achieving energy efficiencies. Very few studies in the past are found to be benchmarked, which is another research gap for which reason, it becomes difficult to access the quality of the techniques being discussed. Also, it was found that algorithm complexity is less concentrated when the techniques are discussed with respect to the performance parameters. Hence, all the above issues are point of focus in the implementation of the proposed model in next section.

\section{PROBLEM IDENTIFICATION}

The problems that are being identified after reviewing the solutions offered in existing system are discussed as follows:

- Few Benchmarked Studies: There are very few benchmarked studies in this problem. It is essential to understand that any solution towards coverage and connectivity should also ensure optimal energy preservation. One means to accomplish this to perform comparative analysis of prior solution to any energy efficient protocols in wireless sensor network. It has been seen that LEACH and its variants offers optimal energy efficiency in wireless network [20]. Surprisingly, very few studies toward coverage and connectivity are found to be compared with LEACH.

- Broader Schemes of Energy Efficiency: It was found that there are multiple techniques for conserving energy e.g. implementing sleep scheduling algorithm [21], selection of cluster heads [22], energy efficient routing schemes [23], minimizing data redundancies [24], etc. However, very few of such techniques were found to be closely associated with addressing coverage and connectivity issues in wireless sensor network.

- Impractical Assumption: Majority of the prior studies in addressing coverage issues have assumed the sensing capability to lie within the sensing area as deterministic [25], which is quite an impractical assumption as it is not possible for a sensor to explore all the sensor range of other sensors in case of large scale network. Also, there are many studies like [26] [27] that have emphasized on cluster head to ensure energy efficiency. However, such schemes have an added flaw as if the cluster head stops working for any particular reason (security, circuitry failure) the connectivity to the other sensor nodes are drastically lost leading to partitioning problems in large-scale sparse network.

\section{PROPOSED SYSTEM}

The prime aim of the proposed system is to present a cost efficient scheme to ensure optimal coverage and connectivity along with energy conservation in wireless sensor network. The case study of the proposed system is experimented with the example of massive data aggregation phenomenon where the formulation is done to ensure that time required for performing data aggregation is not much extensive along with retention of optimal service quality. Table 1 highlights the notation used in empirical discussion of the proposed system.

Table 1. List of Notation Used

\begin{tabular}{llll}
\hline Notation & Meaning & Notation & Meaning \\
\hline$\eta$ & No. of Nodes & $\mathrm{S}_{\mathrm{R}}$ & Sensing Radius \\
$\mathrm{T}_{\mathrm{R}}$ & Transmitting Radius & $(\mathrm{c}, \mathrm{d})$ & $\mathrm{c} \&$ d are $1^{\text {st }}$ and $2^{\text {nd }}$ position of the node \\
$\mathrm{T}_{\mathrm{S}}$ & Transmittance state & $\mathrm{O}_{\mathrm{S}}$ & Off State \\
$\mathrm{S}_{\mathrm{SRS}}$ & Sense/receive state & $\mathrm{S}_{\mathrm{P}}$ & Probability of States \\
$\sigma$ & Anticipated energy used by sensing node & $\mathrm{SIM}_{\text {area }}$ & Simulation Area \\
$\mathrm{S}_{\mathrm{OFF}}$ & Sensing states in off mode & $\mathrm{S}_{\mathrm{i}}$ & Sensing state in i i $^{\text {th }}$ mode \\
$\mathrm{C}_{\text {min }}$ & Minimal Coverage & $\theta$ & Probability of successful transmission \\
$\delta$ & Probability to be retained in $\mathrm{T}_{\mathrm{S}}$ & $\mathrm{N}_{\mathrm{ID}}$ & Node ID \\
$\mathrm{N}_{\text {res_eng }}$ & Node with residual energy information & $\mathrm{d}_{\min }$ & Minimum distance \\
$\mathrm{N}_{\text {member }}$ & Member nodes & & \\
\hline
\end{tabular}

The proposed system models the wireless sensor network into multiple single layered clusters of same sensor nodes of multiple sizes. It was done to facilitate cluster-based communication with the sink in a 
faster manner. Considering this tree-based structure, a communication model is built with specific number of clusters if the network is found to have $\eta$ sensor nodes, where $\eta$ is always greater than the maximum number of nodes in a particular cluster. The system also considers that $\eta$ sensor nodes are structurally approximated by locus that are independently and uniformly distributed in a single shape, $\mathrm{S}_{\mathrm{SRS}}=[0,1] x[0,1]$, where the opposite edges are basically identified. The proposed system adopts the shape of the torus to prevent redundancies owing to edge effects.

\subsection{Addressing Coverage Issues:}

The coverage issues of the wireless sensor network are mitigated by considering the states of the WSN system. We consider the system has equilibrated to its steady state, and every sensor node is treated as independent of the first order with state probabilities represented by $S_{P}$. Consider $S_{R}$ is the sensing radius and $T_{R}$ be the transmitting radius. The coverage model considers that a point $c \in T_{S}$ is said to be covered if there is at least one sensor node in the region with sensing state within $S_{R}$ which will identify the event is occurring at point $c$. Hence, using geometrical approach in the state-based topology, the sensing probability of the particular sensor node can be represented as $\pi \mathrm{S}_{\mathrm{R}}{ }^{2} \mathrm{~S}_{\mathrm{P}}$. Therefore, the probability that no sensor node can sense an event at position $c$ can be represented as $\left(1-\pi \mathrm{S}_{\mathrm{R}}{ }^{2} \mathrm{~S}_{\mathrm{P}}\right)^{\eta}$. Therefore, $\left(1-\pi \mathrm{S}_{\mathrm{R}}{ }^{2} \mathrm{~S}_{\mathrm{P}}\right)^{\eta}$ can be said to be the probability that region $c$ is not have coverage. Hence, using probability theory, the coverage function can be represented as:

$$
f(c)=\left\{\begin{array}{l}
1 c \text { is not covered } \\
0 \text { c is covered }
\end{array}\right\}
$$

Hence, mathematical representation of probability that the position $\mathrm{x}$ is not covered can be represented as $\mathrm{P}[\mathrm{f}(\mathrm{c})=1]=\left(1-\pi \mathrm{S}_{\mathrm{R}}{ }^{2} \mathrm{~S}_{\mathrm{P}}\right)^{\eta}$. The eq.(1) highlights the probability function $f(c)$ that is represented by the conditional criteria for representing covered or uncovered region of wireless sensor network. Therefore, the mathematical representation of the area $\left(\mathrm{SIM}_{\text {area }}\right)$ is not covered up can be depicted as,

$$
S I M_{\text {area }}=\int d c \cdot f(c)
$$

and so $E[A]=\int$ dc. $P[f(c)=1]=\left(1-\pi S_{R}{ }^{2} S_{P}\right)^{\eta}$, where $E\left[S_{\text {area }}\right]$ is expected area that is not covered up. Similarly, mathematical representation of the expected area that is not covered up is 1-E[SIM area $]=1-\left(1-\pi S_{R}{ }^{2} S_{P}\right)^{\eta}$. Hence, the proposed system can easily evaluate the covered area as well as uncovered area in transmission zone in wireless sensor network and can take necessary action. It can also be said that $1-E\left[S_{\text {area }}\right]=1-(1-$ $\left.\pi \mathrm{S}_{\mathrm{R}}^{2} \mathrm{~S}_{\mathrm{P}}\right)^{\eta}$ is going to be the problem space in our study, which is the area that is not covered up and calls for implementing certain techniques to ensure optimal coverage. Considering the fact in this case as $\log (1-c) \leq-c$ for $\mathrm{c}<1$, will provide the scheme as, $\pi \mathrm{S}_{\mathrm{R}}{ }^{2} \mathrm{~S}_{\mathrm{P}}=\sigma(\eta) / \eta$. Then the condition for anticipated coverage is given by,

$$
1-(1-\sigma(\eta) / \eta)^{\eta} \geqslant 1-e^{-\omega \sigma(\eta)}, \text { where } \sigma(\eta) / \eta \leq 1
$$

After assigning the function of coverage and its probability, the area is computed that doesn't come under the coverage area. For the easiness in computation, the expected area to be covered up is computed that will furnish the better probability of the coverage area considered for the study. Thus, as long as $\sigma(\eta) \rightarrow \alpha$ (infinity), the expected coverage approaches $1 . \sigma(\eta)$ can be mapped as the anticipated power used by the sensing nodes. For the purpose of optimization, the proposed system will adopt a technique that will use probability theory for exhibiting the fact that an arbitrary variable will have positive probability of being positive (Second Order Moment). It will also compute variance of area A for which the system should meet the condition of expected area as E[SIM area $]^{2}$. The system also considers average field approximation that the sensor nodes are behaving independently in the simulation area in the presence of neighbor nodes.

\subsection{Addressing Connectivity Issues:}

The proposed system considers possibility of two ideas for ensuring optimal connectivity in the sensor network, as Figure 1. The first idea focuses on the topology of the graph connectivity to be extracted from the availability of the sensor nodes in the network. The second factor proposes more hard condition considering contention issues in network. The proposed system adopts heuristic for mitigating the problems of contention issues in wireless sensor network for ensuring better connectivity solutions. The target of the connectivity can be discussed as follows: Consider an event has occurred in position $c$, where $c \in \mathrm{T}_{\mathrm{S}}$. The communication model will attempt to broadcast this event to next position $d$, where $\mathrm{d} \epsilon \mathrm{T}_{\mathrm{S}}$. Therefore, the 
event message is transmitted from one to another node in multiple positions. The design principle of the proposed study is formulated in such a way that it should support the multi-hop communication system in wireless sensor network. Hence, along with addressing the coverage and connectivity issues, the proposed system also ensure selection of cluster head in the same process. One of the advantages of this process is that the optimal selection of cluster head ensures energy efficient routing in data aggregation process as well as it also ensures optimal connectivity among the cluster.

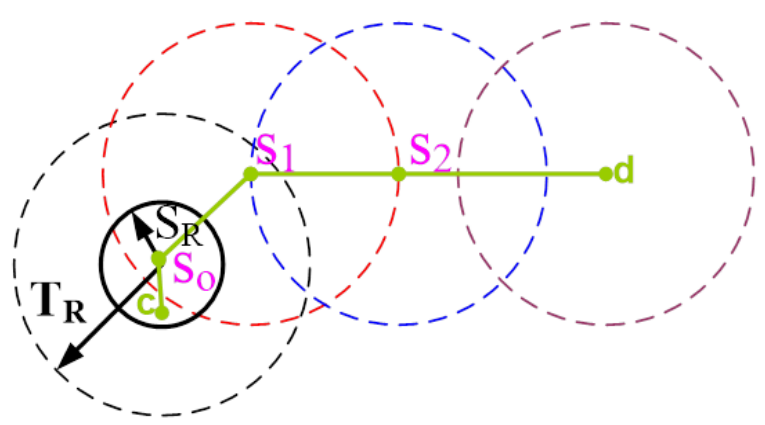

Figure 1. Scenario of coverage and connectivity

The solution to address the connectivity problems in proposed system consists of formulating certain optimal conditions to ensure more robust connectivity among the clusters. The formulation initiates with the fact that source node (in $c$-position) will be transmitting to the destination node (in $d$-position) if there is a presence of multiple hopes in the receiving state at the location $\mathrm{s}_{\mathrm{o}}, \mathrm{s}_{1}, \ldots, \mathrm{s}_{\mathrm{K}}$ such that:

- $\quad \mathrm{P}_{1}$ : It is the first condition of the path setting where $\left|c-\mathrm{s}_{0 \mathrm{FF}}\right| \leq \mathrm{S}_{\mathrm{R}}(c$ can be sensed ;)

- $\quad \mathrm{P}_{2}$ : it is a second condition of the path setting where $\left|\mathrm{s}_{\mathrm{i}}-\mathrm{S}_{\mathrm{i}-1}\right| \leq \mathrm{T}_{\mathrm{R}}$ for $\mathrm{i}=1 \ldots \mathrm{K}$, hence, the event can be transmitted from $\mathrm{s}_{\mathrm{i}-1}$ to $\mathrm{s}_{\mathrm{i}}$, and it will be received since $\mathrm{s}_{\mathrm{i}}$ is in the receiving state.

- $\quad \mathrm{P}_{3}$ : It is the second condition of the path setting where $\left|\mathrm{s}_{\mathrm{K}}-\mathrm{d}\right| \leq \mathrm{T}_{\mathrm{R}}\left(\mathrm{s}_{\mathrm{K}}\right.$ can transmit to $d$ )

Hence, the proposed system supports connectivity for multiple hop (k) network. The system considers condition for minimal connectivity if, for any node $\mathrm{x}$ to node $\mathrm{y}$, there is always existence of at least one edge between them. This solution although mitigates minimal connectivity issues but doesn't mitigate issues related to contention. Hence, it is necessary to formulate a condition for contentionless connectivity for which purpose the proposed system has to ensure that when the node communicates with other node, there should be assurance that the destination node lies within the sensing state, and no other sensor nodes within the neighborhood of destination node should try to perform transmission. If this condition of contention less connectivity is met, redundancies can be controlled which will indirectly influence the energy conservation positively (less redundancies $\rightarrow$ less retransmission $\rightarrow$ energy conservation). Along with connectivity, the proposed system ensures that sensing nodes will require being within coverage area with respect to $S_{R}$. In order to ensure the optimal connectivity, sensing nodes should be covered up in the area with respect to $T_{R}$ as well. Therefore, condition of minimum coverage can be represented as $C_{\min }=\min \left\{S_{R}, T_{R}\right\}$.

\section{IMPLEMENTATION \& RESULT}

The proposed system is implemented on 32-bit Windows OS with $1.84 \mathrm{GHz}$ (min) processor speed, and programming platform is considered in Matlab. The prime purpose of the proposed system is to enhance the coverage and connectivity issues with optimal energy usage in the area of wireless sensor network. The simulation is carried out considering 500-1000 sensor nodes. In order to perform this goal, following the work has been carried out:

- Design of Coverage Enhancement: The implementation for enhancing coverage issue is carried out in dual steps where the first step provides alteration and the second step provides coverage. The first stage evaluates the entire transmission as well as sensing area of a specific sensor node until it converges to a target point. This stage initiates by converting a bigger sensing zone in WSN to modular sensing zone for easiness in computation and extracts various information (residual energy) at the cross-section of its neighbor transmission area which its intersects, so that it can be treated as target in the next consecutive step of coverage enhancement. In order to implement this, the proposed system considers random deployment of sensors in the simulation area of homogenous WSN SIM area, which is wholly covered. The proposed algorithm is executed by all the modular sub-regions of SIM $_{\text {area. }}$ Let $x$ is a transmission area of radius Rad and let another wireless sensor node y be positioned within the range of $x$ and is 
denoted by $\mathrm{y} \epsilon \operatorname{node}(\mathrm{x})$. If the distance between the target Tar in the region and $x$ is less than Rad, that is the distance $(\operatorname{Tar}, x)<$ Rad, Tar is covered by $x$. Let, another node $\mathrm{z}$ be positioned in the communication range $\operatorname{Comm}_{R}$ of $\mathrm{x}$ and is represented as $z \epsilon \operatorname{node}(x)$. The condition proposed here is that of the Euclidian distance between $x$ and $z$ is less than $\operatorname{CommR}$, then they can communicate with each other. The system also assumes that in order to ensure connectivity of the network, the $\operatorname{Comm}_{R}$ of each sensor node must not be smaller than double of its sensing radius Rad.

- Design of Connectivity Enhancement: The enhancement of the connectivity in the proposed system is performed by considering number of set of sensor nodes encapsulating the targets. The implementation of coverage part allocates a cost for every sensor node that illustrates the there are number of targets in the simulation area that needs to be connected to each other. The formulation of the weight considers parameters like residual energy and number of non-encapsulated targets. When choosing a sensor node for a cover set, previous stage signifies on its coverage contribution, that is to say, selecting the one covering as many targets uncovered as possible in this phase, while this phase of design of connectivity considers the tradeoff between power and coverage contribution.

- Power Optimization: The proposed system initially implements a fixed point iteration algorithm to find the stable state probabilities. In addition, the stable state probabilities will depend on -i) the number of nodes in the network, ii) the transmission and sensing radius and iii) the probability of sensing an event, which is an external parameter that depends on the event density. We obtain the steady state by simulating the proposed model to a defined range of simulation rounds and when the node depletes the power.

The proposed system is designed using two algorithms i.e. i) algorithm for energy efficient coverage and connectivity and ii) algorithm to reduce the overhead. The discussions of the formulated algorithms are as follows:

\section{Algorithm for Energy Efficient Coverage and Connectivity \\ Input: Nodes $(\eta)$, Transmission range $\left(T_{X}\right)$, Energy $(E)$ \\ Output: minimum coverage with energy preservation \\ Start}

1. Define simulation parameters $\eta, T_{X}, E$.

2. Formulate probability function for coverage

3. Computed covered area

$$
f(c)=\left\{\begin{array}{l}
1 c \text { is not covered } \\
0 c \text { is covered }
\end{array}\right\}
$$

$$
\pi \mathrm{S}_{\mathrm{R}}^{2} \mathrm{~S}_{\mathrm{P}}
$$

4. Compute Uncovered area

$$
\left(1-\pi \mathrm{S}_{\mathrm{R}}^{2} \mathrm{~S}_{\mathrm{P}}\right)^{\eta}
$$

5. Apply Condition for anticipated coverage

6. Apply Condition of connectivity

$$
1-(1-\sigma(\eta) / \eta)^{\eta} \geqslant 1-\mathrm{e}^{-\omega \sigma(\eta)}, \text { where } \sigma(\eta) / \eta \leq 1
$$

$\left|\mathrm{c}-\mathrm{S}_{0 \mathrm{FF}}\right| \leq \mathrm{S}_{\mathrm{R}}$

$\left|\mathrm{S}_{\mathrm{i}}-\mathrm{S}_{\mathrm{i}-1}\right| \leq \mathrm{T}_{\mathrm{R}}$ $\left|\mathrm{s}_{\mathrm{K}}-\mathrm{d}\right| \leq \mathrm{T}_{\mathrm{R}}$

7. Define three state metric of energy $E_{0}, E_{S}, E_{T}$.

8. Compute anticipated energy depletion in steady state: $\mathrm{E}=\left(\mathrm{E}_{\mathrm{o}} \sigma_{\mathrm{o}}+\mathrm{E}_{\mathrm{S}} \sigma_{\mathrm{S}}+\mathrm{E}_{\mathrm{T}} . \sigma_{\mathrm{T}}.\right) / / \sigma_{\mathrm{o}}, \sigma_{\mathrm{S}}, \sigma_{\mathrm{T}}$ are anticipated energy in $\mathrm{T}_{\mathrm{o}}, \mathrm{O}_{\mathrm{S}}$, and $\mathrm{S}_{\mathrm{SRS}}$.

9. Define transmission probability:

$$
\theta=\theta_{1} /\left(1-\delta+\delta \theta_{1}\right)
$$

10. Apply minimization function $\operatorname{argmin}\left(\mathrm{E}_{\mathrm{o}} \mathrm{p}_{\mathrm{o}}+\mathrm{E}_{\mathrm{S}} \mathrm{p}_{\mathrm{S}}+\mathrm{E}_{\mathrm{T}} \cdot \mathrm{p}_{\mathrm{T}}\right)$

11. Evaluate the minimum coverage

\section{End} $\mathrm{r}=\min \left\{\mathrm{r}_{\mathrm{s}}, \mathrm{r}_{\mathrm{T}}\right\}$

In the above algorithm, $\theta$ is the probability of successful data packet transmission, and $\theta_{1}$ can be represented as probability of the initial successful data transmission attempt. $\delta$ is the assumption of probability to be retained in the transmission state. As discussed in the previous section that the proposed system perform selection of cluster head for optimizing the energy by reducing the overhead owing to contention. The 
optimization is done by estimating the overhead of energy consumed by the communication module with following steps e.g.

Algorithm for reducing overhead

Input: $\mathrm{N}_{\mathrm{ID}}, \mathrm{T}_{\mathrm{R}}, \mathrm{SIM}_{\text {area }}, \mathrm{N}_{\text {res eng. }}$.

Output: Unique fusing of data packets with less energy

Steps:

\section{Start}

1 Initialize simulation area;

2 Initialize Tree $\left[\mathrm{F}_{\mathrm{T}}:\left\{\mathrm{N}_{\mathrm{ID}}, \mathrm{T}_{\mathrm{x} \_ \text {Range }}, \operatorname{Sink}_{\mathrm{x}, \mathrm{y}}\right\}\right]$;

3 Estimate Sink-location:

$$
\text { Sink } k_{x}=\text { Area }+B_{\text {dist }} \quad \text { Sink }_{y}=\text { Area }+B_{\text {dist }}
$$

4 Initialize Neighborhood density $\left(\mathrm{ND}_{\max }=N-1\right)$.

5 Perform Energy based sorting of nodes

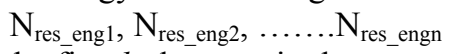

6 Select the first $k$ elements in the set as the CHS of networks.

$$
\mathrm{K}=\left\{\mathrm{CH}_{1}, \mathrm{CH}_{2}, \mathrm{CH}_{3}, \mathrm{CH}_{4}, \mathrm{CH}_{5}\right\}
$$

7 Select member node with minimum distance

$$
\mathrm{N}_{\text {member }} \rightarrow \mathrm{d}_{\text {min }}
$$

8. Compute location of $\mathrm{CH}$

9. Calculate the distance from all other nodes

10. Decide the number of member for every cluster head, and

Cardinal $\left(\mathrm{N}_{\text {member node }}\right) \rightarrow \mathrm{CH}$

11. Perform communication to respective cluster head.

\section{End}

The proposed system is implemented in Matlab considering the case study of multiple cycles of coverage and connectivity problems. Each of the event nodes can generate an event with a certain firing probability. The firing probability is related to the user input sensing the probability.

It can be seen from the results that its transceiver module consumes most of the energy in a wireless sensor node. The objective of a network is to extend the coverage and connectivity mechanism from all of its sensor nodes. Each sensor node was initialized by a random residual energy level. A network was assessed based on its packet delivery ratio (Figure 1) and total power consumption (Figure 2) and the duration of an attempt to increase connectivity.

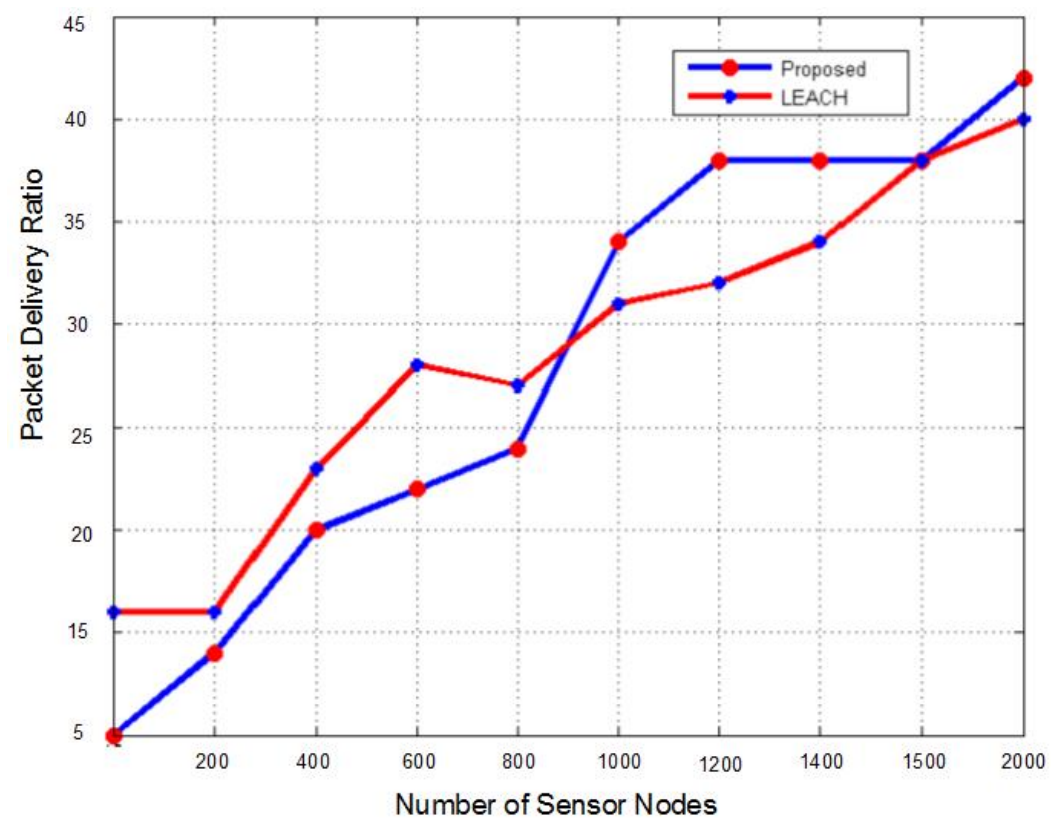

Figure 2. Packet Delivery Ratio 
To investigate the effect of in-network coverage and connectivity to the performance of a network, different values of radius were considered in the simulations. For comparison purposes, frequently used LEACH algorithm [28] is considered. As expected, both packet delivery ratio and total energy optimization increase with the number of nodes, regardless of radius and network structures. When comparing the total energy consumed in an attempt to extend the coverage and connectivity process in WSN, the performance of networks with the proposed network structure is better than those with LEACH. Despite the obvious advantages of using LEACH protocol for cluster organization, few features are still not supported. LEACH assumes a homogeneous distribution of sensor nodes in the given area. This scenario is not very realistic. Another biggest problem with the LEACH algorithm is cluster head selection is done quite randomly for which reason, the core nodes are very fast depleted of energy. The proposed algorithm does the energy management using enhancement design for coverage and connectivity. The proposed system also ensures that while coverage and connectivity are enhanced, it shouldn't have any negative influence on its energy / lifetime of the nodes.

It is quite evident from Figure 2 and Figure 3 that the proposed system has successfully derived an algorithm that can perform energy efficient coverage and connectivity. The proposed system performs cumulative energy optimization along with enhancement of coverage and connectivity level. Figure4 illustrate the analysis of connectivity level of the proposed system, where it can be seen that proposed system can maintain smooth ascent in the curve representing spontaneous data transmission process. Hence, the connectivity level is found with maximum ascent along with energy efficiency for proposed system as compared to the LEACH algorithm

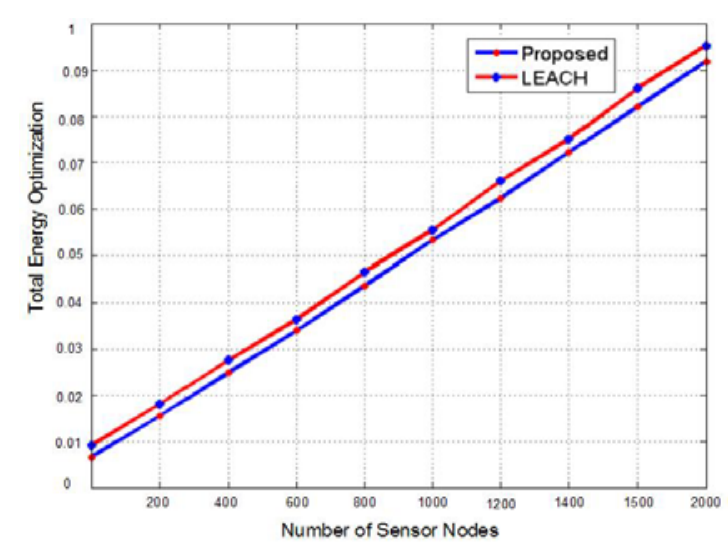

Figure 3. Total Energy Optimization

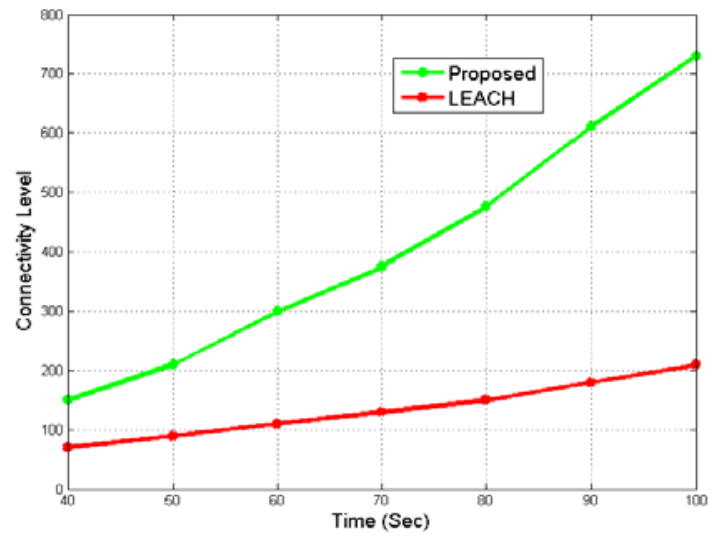

Figure 4. Analysis of Connectivity Level

Figure 5 represents the analysis of the coverage level, where it can be seen that proposed system is found with maximum coverage level as compared to LEACH algorithm.

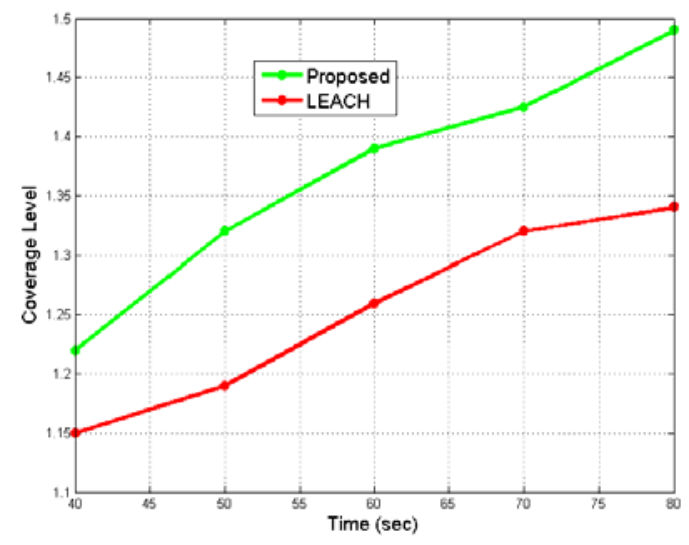

Figure 5. Analysis of Coverage Level

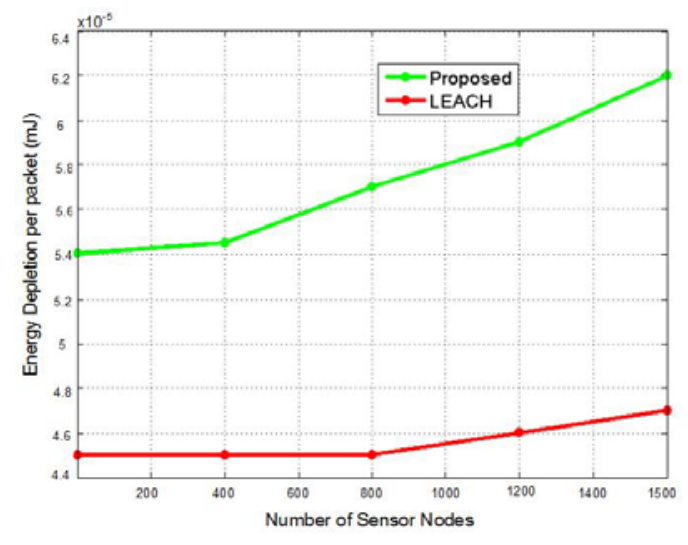

Figure 6. Analysis of Energy Depletion per packet (mJ) 
Figure 6 represents the analysis of the energy consumption per packet $(\mathrm{mJ})$. Interestingly, the proposed system extends the coverage and connectivity level along with energy optimization and is found to be of superior design (with respect to energy) compared to LEACH protocol. Figure 6 is a micro level analysis for Figure 3, where the framework cumulatively ensures better strategies for enhancing issues pertaining to coverage and connectivity. Hence, the proposed system offers a simple and efficient coverage and connectivity solutions for large-scale wireless sensor network.

\section{CONCLUSION}

The proposed system highlights optimization process that is designed to construct the proposed network structure, which helps maintaining the total energy consumption at a low level. Simulation results show by using the proposed model, the packet delivery ratio, and cumulative energy optimization has been increased. The entire policy of designing enhancement of coverage and connectivity issues in WSN is based on a probabilistic approach. The proposed system has used an enhancement policy that targets to preserve cumulative energy consumption and addresses extending connectivity too by a probabilistic method of turning off all the redundant sensor nodes

\section{REFERENCES}

[1] I.M.M. El Emary, S. Ramakrishnan, "Wireless Sensor Networks: From Theory to Applications", CRC Press, Computers, Aug 2013.

[2] H.M. Ammari, "The Art of Wireless Sensor Networks", Springer Science \& Business Media, Computers, Dec 2013.

[3] G. Anastasi, M. Conti, M.D. Francesco, A. Passarella, "Energy Conservation in Wireless Sensor Networks: a Survey”, Elsevier-Adhoc Networks, Vol. 7, Iss. 3, pp. 537-568, May 2009.

[4] M.A. Guvensan, A.G. Yavuz, "On coverage issues in directional sensor networks: A survey", Elsevier- Ad Hoc Networks, vol. 9, pp. 1238-1255, Sep 2011.

[5] A.Nikdel, M.S. Kohshoori, and S.M. Jamei, "An Intelligent and Energy Efficient Area Coverage Protocol for Wireless Sensor Networks", International Journal of Grid and Distributed Computing, Vol. 4, No. 4, Dec 2011.

[6] L. Sharma, J.Singh, S. Agnihotri, "Connectivity and Coverage Preserving Schemes for Surveillance Applications in WSN", International Journal of Computer Applications, Vol. 50, No. 19, July 2012.

[7] D.S. Sakkari, T.G. Basavaraju, "Extensive Study on Coverage and Network Lifetime Issues in Wireless Sensor Networks", International Journal of Computer Applications, Vol. 52, No. 8, Aug 2012.

[8] S.A.R. Zaidi, M. Hafeez, S.A. Khayam, D.C. Mclernon, M. Ghogho, and K. Kim, "On Minimum Cost Coverage in Wireless Sensor Networks", IEEE-Annual Conference on Information Sciences and Systems, pp. 213-218, Mar 2009.

[9] P.K. Agarwal, E. Ezra, and S. Ganjugunte, "Efficient Sensor Placement for Surveillance Problems", Proceedings of $5^{\text {th }}$ IEEE Conference on Distributed Computing in Sensor Networks, pp. 301-314, Aug 2009.

[10] E. Bulut, Z. Wang and B.K. Szymanski, "The Effect of Neighbor Graph Connectivity on Coverage Redundancy in Wireless Sensor Networks", IEEE International Conference on Communications, pp. 1-5, May 2010.

[11] J. Beaudaux, A. Gallais, T. Razafindralambo, "Multiple Coverage with Controlled Connectivity in Wireless Sensor Networks", Proceedings of the 7th ACM workshop on Performance evaluation of wireless ad hoc, sensor, and ubiquitous networks, pp. 9-16, Oct 2010.

[12] P. Wang, Z.Sun, M.C. Vuran, M.A. Al-Rodhaan, A.M. Al-Dhelaan, I.F. Akyildiz, "On network connectivity of wireless sensor networks for sandstorm Monitoring”, Elsevier-Computer Networks, vol. 55, pp. 1150-1157.

[13] E. Kranakis, D. Krizanc, A. Modi, O. M.Ponce, "Connectivity Trade-offs in 3D Wireless Sensor Networks using Directional Antennae", IEEE-International Parallel and Distributed Processing Symposium, pp. 345-351, May 2011.

[14] Y.Stergiopoulos, Y. Kantaros, and A. Tzes, "Distributed Control ofMobile Sensor Networks under RF Connectivity Constraints", Hindawi Publishing Corporation International Journal of Distributed Sensor Networks, Article ID 741821, doi:10.1155/2012/741821, 2012.

[15] M. Erdelj, V. Loscri, E. Natalizio, T. Razandralambo, "Multiple Point of Interest Discovery and Coverage with Mobile Wireless Sensors", Elsevier-Ad Hoc Networks, vol. 11, Iss. 8, pp. 2288-2300, 2013.

[16] X. Ren, W. Liang, W. Xu, "Quality-Aware Target Coverage in Energy Harvesting Sensor Networks", IEEETransaction on Emerging Topics in Computing, Iss. 99, DOI: 10.1109/TETC.2014.2371543, 2014.

[17] H. Noori and R. Rafeh, "A novel intelligent Sleep Wakeup Scheduling algorithm to the Area Coverage problem in Wireless Sensor Networks”, International Journal of Grid and Distributed Computing, Vol. 7, No. 1, pp. 53-66, Sep 2014.

[18] H.Zhang and J.C. Hou, "Maintaining Sensing Coverage and Connectivity in Large Sensor Networks", Ad Hoc \& Sensor Wireless Networks, Vol. 1, pp. 89-124, Mar 2005.

[19] F. Taymouri, M.R. kangavari, "Measuring Connectivity Tolerance in Wireless Sensor Networks using Graph Theory Applications: A Fast Algorithm", International Journal of Scientific and Engineering Research, Vol. 5, Iss. $2,2014$. 
[20] V. Kumar, S. Jain, and S. Tiwari, "Energy Efficient Clustering Algorithms in Wireless Sensor Networks: A Survey”, International Journal of Computer Science Issues, Vol. 8, Issue 5, No 2, Sep 2011.

[21] E. Bulut, I. Korpeoglu, "Sleep Scheduling with Expected Common Coverage in Wireless Sensor Networks", $A C M$ Journal of Wireless Network, vol. 17, Iss. 1, Jan 2011.

[22] M. Lehsaini, H. Guyennet, and M. Feham, "CES: Cluster-based Energy-efficient Scheme for Mobile Wireless Sensor Networks, International Federation for Information Processing", Springer-Wireless Sensor, and Actor Networks II, Vol. 264, pp. 13-24, Jul 2008.

[23] S. Singh, Meenaxi, "A Survey on Energy Efficient Routing in Wireless Sensor Networks", International Journal of Advanced Research in Computer Science and Software Engineering, Vol. 3, Iss. 7, Jul 2013.

[24] H. Luo, Y. Liu and S.K. Das, "Routing Correlated Data in Wireless Sensor Networks: A Survey", IEEE Network, vol. 21, Iss. 6, pp. 40-47, Dec 2007.

[25] R. Mulligan, "Coverage in Wireless Sensor Networks: A Survey", Network Protocols and Algorithms, ISSN 19433581 2010, Vol. 2, No. 2, 2010.

[26] M. Maimour, H. Zeghilet and F. Lepage, "Cluster-based Routing Protocols for Energy Efficiency in Wireless Sensor Networks", Intech-Open Science, DOI: 10.5772/13274, Dec-2010.

[27] V. Sasikala and C. Chandrasekar, "Energy Efficient Multipath Data Fusion Technique for Wireless Sensor Networks", ACEEE International Journal on Network Security, Vol. 03, No. 02, Apr-2012.

[28] W.R. Heinzelman, A. Chandrakasan, and H. Balakrishnan, "Energy-Efficient Communication Protocol forWireless Microsensor Networks", IEEE-Proceedings of the 33rd Hawaii International Conference on System Sciences 2000 .

\section{BIOGRAPHIES OF AUTHORS}
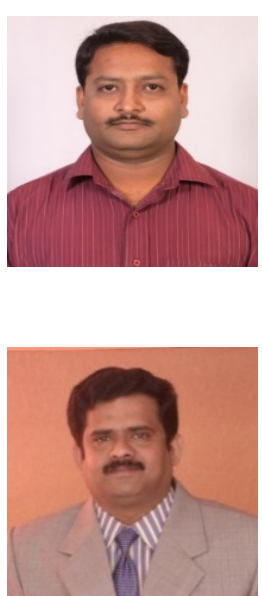

Deepak S Sakkari received B.E degree in Instrumentation \& Electronics Engineering from Bangalore University, Karnataka, India. M. Tech Degree in Information Technology from AAIDU, Allahabad. He is now pursuing his Ph.D. degree from Jawaharlal Nehru Technological University, Hyderabad, Andhra Pradesh. His research interest includes Wireless Sensor Networks especially the Coverage and Lifetime Optimization in Wireless Sensor Networks. He is currently working as Assistant Professor in the Department of Computer Science \& Engineering of Acharya Institute of Technology, Bangalore, Karnataka.

Dr. T G Basavaraju is currently working as Professor and Head of Computer Science and Engineering Department at GovtSKSJ Technological Institute, Bangalore. Prof.Basavaraju holds a Ph.D. (Engg.) from Jadavpur University, Kolkata in the area of Mobile Ad hoc Networks. He obtained his Master's Degree in Computer Science and Engineering from University Visvesvaraya College of Engineering (UVCE), Bangalore University, Bangalore and secured first rank. He holds Bachelor's degree in Computer Science and Engineering from University BDT College of Engineering (UBDTCE), Kuvempu University, Davangere. He has more than 16 years of experience in Teaching and Industry. He has authored and co-authored five textbooks in the area of Computer Networking. One of his co-authored textbook on" Mobile Wireless Ad hoc Networks: Principles, Protocols, and Applications" was published by Auerbach Publishers (Taylor and Francis group), USA. His major areas of research are Wireless Ad hoc Networks, Sensor Networks, and Mesh Networks. He has to his credit more than 45 research publications in National/International Journals and Conferences. 